Review

\title{
Migraine as a Common Extra-Intestinal Presentation of Celiac Disease
}

\author{
Maurizio Gabrielli* , Veronica Ojetti, Marcello Candelli, Luca Santarelli, Antonio Gasbarrini, \\ Francesco Franceschi \\ Fondazione Policlinico Universitario A. Gemelli IRCCS, Università Cattolica del Sacro Cuore \\ Department of Emergency Medicine, Rome, Italy; E-Mails: maurizio.gabrielli@policlinicogemelli.it; \\ veronica.ojetti@unicatt.it; marcello.candelli@policlinicogemelli.it; \\ luca.santarelli@policlinicogemelli.it; antonio.gasbarrini@unicatt.it; \\ francesco.franceschi@unicatt.it
}

* Correspondence: Maurizio Gabrielli; E-Mail: maurizio.gabrielli@policlinicogemelli.it

Academic Editor: Luis Rodrigo

Special Issue: Gluten-Related Neurological Disorders

OBM Neurobiology

2021, volume 5, issue 1

doi:10.21926/obm.neurobiol.2101087
Received: August 18, 2020

Accepted: January 18, 2021

Published: February 09, 2021

\begin{abstract}
Celiac disease $(C D)$ is a common gluten-related enteropathy that presents several extraintestinal manifestations. Migraine is globally recognized as one of the most diffuse primary headaches. The present paper aims to review the current evidence on the possible association between $C D$ and migraine. Both adults and children having CD exhibit a high prevalence of migraine. However, screening for $C D$ in an unselected population of migraineurs demonstrated contrasting results. Brain imaging may reveal cerebral calcifications and/or white matter abnormalities in a small number of patients having migraine with underlying $C D$. Limited data are available on the effects of gluten-free diet (GDF) on migraine in the patients affected by $C D$, suggesting the efficacy of such intervention on the migraine symptoms in both adults and children. Nonetheless, this inference requires verification with high-quality trials conducted with larger sample sizes and longer follow-up periods.
\end{abstract}

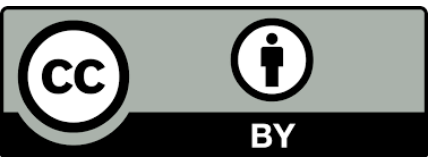

(C) 2021 by the author. This is an open access article distributed under the conditions of the Creative Commons by Attribution License, which permits unrestricted use, distribution, and reproduction in any medium or format, provided the original work is correctly cited. 


\section{Keywords}

Celiac disease; headache; migraine

\section{Introduction}

Celiac disease $(C D)$ is a peculiar disorder that manifests as an autoimmune enteropathy triggered by gluten ingestion in genetically predisposed individuals [1]. Tissue transglutaminase is the auto-antigen involved in CD, while human leukocyte antigen (HLA)-DQ2 and HLA-DQ8 are the key genetic elements [1]. The positivity of serological tests is suggestive of CD. The anti-tissue Transglutaminase (anti-tTG) and anti-Endomysium antibodies (EmA) exhibit higher diagnostic accuracy compared to deamidated gliadin peptide antibodies, which are currently used for identifying $C D$ in early childhood (age $<2$ years) [1]. However, the gold standard for CD diagnosis is the duodenal biopsy that reveals the mucosal damage of the small intestine [ranging from minimal lesions that are characterized by an isolated increase in the intraepithelial lymphocytes to complete villous atrophy] [1].

$\mathrm{CD}$ is one of the most common autoimmune disorders worldwide. According to a recent metaanalysis, the pooled global prevalence of $C D$ is $1.4 \%$ when determined using serologic screening and $0.7 \%$ when determined using duodenal biopsy. The prevalence is slightly higher in Europe and Oceania compared to the other continents. In addition, the disorder is more prevalent in females than in males. The rates of initial diagnosis are higher in children compared to the adult population [2].

The immune-inflammatory process is reported to specifically affect the gut mucosa, although its presentation is extremely variable and ranges from different gastrointestinal symptoms to various extra-intestinal symptoms [3]. Migraine is reported as one of the most common neurological diseases associated with $C D$, both in infants and adults [3-5].

Second, only to the tension-type headache, migraine is the most prevalent among the primary headaches on a global scale. The global prevalence of migraine is $11.6 \%$ (10.4\% in Africa, $10.1 \%$ in Asia, $11.4 \%$ in Europe, $9.7 \%$ in North America, and $16.4 \%$ in Central and South America). In adults, migraine occurs mostly in the age range of 25-55 years, with females being significantly more affected compared to males. In young children, migraine is the most common subtype of recurrent headache [6], while in late childhood, migraine is the second most frequent (9\%) among the primary headaches after the tension-type headache [6].

The present report aimed to review the literature to find the existing pieces of evidence for the possible association between $C D$ and migraine. In order to achieve this objective, a literature search was performed on two databases/search engines-PubMed and Google Scholar-until August 2020, using the following search string: ("Celiac disease" or "Gluten") and ("Migraine" or "Headache" or "Neurological"). In addition, a manual search was conducted by examining the bibliography provided in every included study and the related references. All the research papers identified in these two search steps were evaluated in relevance to the topic of concern, and the clinically significant articles were selected for inclusion in the present review. 


\section{Prevalence of Migraine in both Adult and Pediatric Patients with Celiac Disease}

According to a large retrospective population-based study conducted by Lebwohl et al., headache-related health care visits were significantly more common in patients with $C D$ in comparison to controls (4.7\% vs. $2.9 \%$ ) [7].

In a meta-analysis conducted in 2018, which included five cohort and four case-controlled studies, a pooled mean prevalence of headache in the adults with CD was reported to be $26 \%$ (95\% $\mathrm{Cl}$ 19.5-33.9\%), with the odds of experiencing a headache significantly higher in the CD patients compared to controls (OR 2.7, 95\% Cl 1.7-4.3, p < 0.0001) [8]. However, none of the above-stated studies provided information regarding the headache subtypes.

An online survey promoted on the Argentinean Celiac social networks investigated the characteristics of the headache in celiac patients, with a total of 866 individuals fulfilling the inclusion criteria of headache and CD confirmed with a positive biopsy. It was revealed that the tension-type headache was the most prevalent headache type (52\%), followed by migraine without aura (32.5\%) and with aura (15.4\%), respectively. Interestingly, $24 \%$ of the subjects included in this survey reported headache as the main symptom leading to the diagnosis of CD [9].

Only two case-control studies on the topic of concern are available in the literature. In one of these studies, $28 \%$ of the 72 adult patients with biopsy-proven CD presented migraine as one of the neurological symptoms [10]. In another study, Dimitrova et al. reported that 40 patients among the 188 patients with CD had a concomitant migraine, in contrast to only 13 patients among the 178 controls $(O R=3.79)$. The authors reported that this association was stronger in women and those aged $<65$ years [11]. In addition, a trend toward more severe headaches in CD patients compared to the controls was observed by the authors.

In regard to the pediatric population, the meta-analysis conducted by Zis et al. reported an $18.3 \%$ (95\% Cl 10.4\%-30.2\%) pooled mean prevalence of primary headaches in children and adolescents with $C D$ [7]. In regard to migraine specifically, the data reported for pediatrics were similar to those observed in adults. In a large, cross-sectional, population-based study conducted on Israeli adolescents (median age of 17.1 years), the multivariate analysis revealed that migraine was significantly more common in CD patients compared to controls (OR 2.3) [12].

Two retrospective studies reported 18.6\% (16 among 86 subjects) and 25\% (33 among 132 subjects) prevalence of migraine among the children with $C D[13,14]$.

Case-control studies on the topic of concern are also available in the literature, with a significantly higher prevalence of migraine reported in CD patients compared to controls $(12.6 \%$ among 111 subjects versus $5.7 \%$ among 211 subjects) [15].

\section{Prevalence of Celiac Disease in both Adult and Pediatric Patients with Migraine}

A few case reports have suggested migraine as the possible first presentation of CD [16-20]. In a study conducted by our group with 90 adults having migraine and the 236 sex-matched and agematched controls, CD (screened using anti-tTG and EmA, and the positive cases confirmed by biopsies) was diagnosed in $4.4 \%$ of the patients with migraine and $0.4 \%$ of the controls $(p<0.05)$ [21]. No other data are available regarding the screening for $C D$ in the adults affected by migraine.

Relatively greater evidence is available from the pediatric population. In a study by Lahat et al., it was observed that the anti-Endomysial antibodies were negative in all 41 children with migraine 
headache (1-16 years of age) [22]. Five related case-control studies are available in the literature [23-27], two of which, both conducted with the Turkish population, reported negative findings [23, 24]. In one of these studies, no difference was observed in the positivity of anti-tTG between the migraine patients and the healthy controls ( $2 \%$ in both; $2 / 100$ versus $30 / 1500$, respectively) [23]. The other study, conducted by Balci et al., reported similar results, with 2 among the 81 patients having migraine exhibiting positivity for anti-tTG antibodies compared to 1 among the 176 controls $(p=n s)$. In the same study, the duodenal biopsy was negative in all the subjects, who were then considered 'potential celiacs' [24].

In another Turkish study conducted with children in the age range of 6-17 years, a significantly higher prevalence of positive anti-tTG antibodies was reported in the 73 patients with migraine $(5.5 \%)$ compared to the 147 controls $(0.6 \%, p<0.05)$. However, the patients with positive serology exhibited normal duodenal histology, suggesting a condition of 'potential CD' [25].

On the other hand, two case-control studies concerning the Italian population reported a positive association between migraine and CD. Lionetti et al. reported that 4 among the 79 (5\%) patients with primary headaches had $C D$. The prevalence was significantly higher compared to the $0.6 \%$ prevalence observed in the general pediatric population in the same geographical area. Jejunal biopsy confirmed the diagnosis of $C D$ in all children included in the study [26]. It is noteworthy that, in this study, the patients with a combined diagnosis of migraine and tension headaches were enrolled, as according to the authors, a clear distinction between these two forms of headache in children and adolescents was difficult and often confusing [28].

In a larger study, Nenna et al. screened 883 children (481 females, median age 9.8 years) attending a tertiary care Centre for Pediatric Headache for the presence of CD. Among these children, eighteen $(18,2.04 \%)$ received a final diagnosis of CD (positive serology confirmed by duodenal biopsy). The prevalence was significantly higher compared to the $1.2 \%$ prevalence observed in the general population $(p=0.034)$. The most common type of headache observed in this sample was migraine (without aura 50\%, and with aura $11 \%$ ), followed by tension-type headache and migraine with aura (38.9\%) [27].

\section{Imaging in Migraine and Celiac Disease}

Computed tomography (CT) is usually a norm in primary headaches, including migraine headaches. Interestingly, a few case reports demonstrate that newly diagnosed CD, migraine-like headache, and occipital or parieto-occipital calcifications in adults and children are well evident in CT scans $[8,16,19,29]$. In certain pediatric cases, cerebral calcifications were associated with both migraine and epilepsy, thereby resembling what is referred to as the 'epilepsy and cerebral calcification syndrome' [8]. The association between these two diseases could be based on the "vascular" pathophysiological mechanism. Indeed, the pathological specimens exhibit various cortical vascular abnormalities, such as venous hemangiomas in deep cortical layers, subcortical calcification of the vessel walls, and large jagged microcalcifications, among others [30]. Such abnormalities are similar to but not identical with those observed in Sturge-Weber syndrome, another condition with the possible coexistence of migraine and epilepsy [31].

Non-specific white matter abnormalities (WMA) detected in magnetic resonance imaging (MRI) could be an incidental finding in normal control populations, although these are significantly more common in individuals with cerebrovascular risk factors [32]. An increased rate of WMA was 
reported in migraine patients [33], suggesting a risk of future cerebrovascular events such as ischemic stroke [34]. Interestingly, WMA are common in CD patients, particularly those with concomitant migraine. A study reported 12 among the 33 adults (36\%) with CD and neurological comorbidities to have WMA compared to none among the controls [35]. The strongest association was observed in the sub-group with migraine-like headache, with four among the six patients exhibiting WMA. Moreover, this category also exhibited the greatest number of WMA per scan. No major cardiovascular risk factor was present in these patients. Similar findings were reported by Hadjivassiliou et al., who observed that all four subjects with episodic migraine-like headache and CD exhibited WMA [36]. Only one study concerning children is available in the literature, and it reports the presence of WMA in one among the four patients with positive anti-tTG serology [25].

The etiology of WMA in CD remains unknown so far, although an immune-mediated vasculitis is suggested [35]. The common detection of WMA in patients with migraine and CD, particularly in adults, suggests that this category might be at a higher risk of future cardiovascular disease, including ischemic heart disease, ischemic stroke, and vascular dementia [37, 38].

In regard to Single Photon Emission Computed Tomography (SPECT) imaging, the available data are from a study conducted with two groups of patients, one with four adults having migraine and untreated $C D$ and the other with five controls having migraine without $C D$, involving the administration of $740 \mathrm{MBq}$ of $99 \mathrm{mTc}$ hexemethyl-propylene-amineoxime in a headache-free period. All the patients in the first group exhibited evident abnormalities in regional cerebral blood flow, which was characterized by a circumscribed area of cortical hypoperfusion. In contrast, no interhemispheric asymmetries of cortical regional blood flow were observed in the group of controls [21]. Interestingly, complete disappearance of the SPECT abnormalities was observed at the 6-month follow-up performed during the gluten-free diet (GFD) period.

\section{Effect of Gluten-Free Diet in Adults and Pediatrics}

Currently, the data on the effect of GFD on migraine symptoms are scarce. It is reported that after commencing GFD, the migraine disappears or significantly improves only in a proportion of CD patients [16-20].

In a small series of adult patients with both CD and migraine, GFD was reported to be associated with the disappearance of headache in one subject and a marked improvement in the intensity, frequency, and duration of attacks in the other subjects [21].

Nenna et al. reported a successful GFD, which resulted in a significant improvement in the headache symptoms in all 18 children affected with a recurrent primary headache that was diagnosed as CD after the serological screening and histological confirmation [27].

The other cohort studies assessing the effect of GFD on headache in adult and pediatric CD patients $[10,11,39]$ did not distinguish the results according to migraine or the other subtypes [15, $26,40,41]$, and all these studies reported a substantial amelioration of headache in a substantial proportion of patients.

Since all these studies were uncontrolled, the improvement observed in the migraine symptoms upon using GFD might be a placebo effect. However, it is noteworthy that placebo is reported to have a strong influence on acute migraine (up to $75 \%$ response in symptoms scores), 
while its effect on chronic migraine remission is estimated to be less significant (approximately 25\%) [42].

\section{Possible Pathophysiological Mechanisms}

Several mechanisms have been proposed for the association between migraine and $C D$, although none of these has been definitively proven [43-48]. Migraine is a neurovascular condition characterized by the dysfunction of cerebral nerves and blood vessels. Calcitonin gene-related peptide (CGRP) is reported to be strongly involved in the pathophysiology of migraine [43-45]. Migraine-specific triggers cause the dilation of cranial blood vessels, which activates the perivascular trigeminal sensory nerve fibers, ultimately resulting in the release of vasoactive peptides, such as substance $P$ and particularly CGRP. These peptides are reported to exacerbate vasodilation and cause neurogenic inflammation that is characterized by vasodilation, leakage of blood vessels, and degranulation of mast cells [36-38]. Pro-inflammatory cytokines, such as tumor necrosis factor-alpha and interferon-gamma, typically released in CD patients after gluten ingestion, may promote, via complex mechanisms, the synthesis of CGRP, thereby triggering migraine attacks [43-45].

An alternative hypothesis states that the malabsorption associated with CD may lead to a deficiency of vitamins and macro-elements (magnesium), thereby affecting migraine [19, 43]. In particular, folate deficiency, which is known to increase the plasma levels of homocysteine, could play a role in the cerebral vascular dysfunction typical of migraine, as well as in the development of cerebral calcifications detected in certain cases of migraine associated with $\operatorname{CD}[16,19]$.

$C D$ is an autoimmune disease associated with several other autoimmune disorders, and therefore, migraine in $C D$ patients could be the consequence of the neurological damage mediated by anti-neuronal and anti-ganglioside antibodies, which are detected frequently in the CD patients, particularly those with neurological disorders, with cerebellar ataxia and epilepsy being the most common ones [46-48]. These autoantibodies tend to disappear after a strict gluten-free diet, as reported in certain studies [46, 47]. Although no data confirming this inference are available in the literature concerning patients with $C D$ and migraine, it remains a plausible mechanism, recalling the autoimmune hypothesis of WMA in CD.

\section{Discussion and Conclusion}

In an attempt to summarize the current pieces of evidence regarding the association between migraine and $C D$, the following key points are highlighted:

a. According to the data in the literature, there is a high prevalence of migraine in CD patients, both adults and children.

b. On the contrary, the screening for CD using serological tests in an unselected patient population with migraine remains debatable due to the conflicting findings of the reported studies. The screening may probably be justified when the other findings also suggest CD diagnosis (i.e., gastrointestinal symptoms, iron-deficiency-based anemia, and autoimmune disorders typically associated with $C D$ ) or when cerebral calcifications and/or WMA are revealed in brain imaging.

c. Preliminary data suggest that GFD may improve the intensity, duration, and frequency of headache attacks in patients with the migraine associated with CD. Considering the importance of GFD in treating the CD enteropathy and the majority of the associated extra-intestinal disorders, it 
is recommended to motivate the patients to strictly follow the prescribed GFD. In addition, the physicians should strictly monitor their patients and conduct periodical serological testing to assess their compliance with GFD.

d. Although the available literature suggests that migraine should be considered a common extra-intestinal disorders associated with $C D$, further studies with larger sample sizes, in both adults and children, that assess the prevalence of CD in migraine and the effects of GFD on migraine attacks (possibly with a consistent follow-up) are warranted to confirm this suggestion. Since several of the published trials did not include a proper definition of the diagnosis of primary headache, future trials should take this into consideration. In addition, future studies should focus on unraveling the mechanisms underlying the association of $C D$ and migraine.

\section{Author Contributions}

Maurizio Gabrielli and Francesco Franceschi conceived and wrote the paper; Veronica Ojetti, Marcello Candelli and Luca Santarelli analyzed and collected all the available literature; Francesco Franceschi and Antonio Gasbarrini revised the draft of the manuscript.

\section{Competing Interests}

The authors have declared that no competing interests exist.

\section{References}

1. Caio G, Volta U, Sapone A, Leffler DA, De Giorgio R, Catassi C, et al. Celiac disease: A comprehensive current review. BMC Med. 2019; 17: 142.

2. Singh P, Arora A, Strand TA, Leffler DA, Catassi C, Green PH, et al. Global prevalence of celiac disease: Systematic review and meta-analysis. Clin Gastroenterol Hepatol. 2018; 16: 823836.e2.

3. Leffler DA, Green PH, Fasano A. Extraintestinal manifestations of coeliac disease. Nat Rev Gastroenterol Hepatol. 2015; 12: 561-571.

4. Arzani M, Jahromi SR, Ghorbani Z, Vahabizad F, Martelletti P, Ghaemi A, et al. Gut-brain Axis and migraine headache: A comprehensive review. J Headache Pain. 2020; 21: 15.

5. Woldeamanuel YW, Cowan RP. Migraine affects 1 in 10 people worldwide featuring recent rise: A systematic review and meta-analysis of community-based studies involving 6 million participants. J Neurol Sci. 2017; 372: 307-315.

6. Wöber-Bingöl Ç. Epidemiology of migraine and headache in children and adolescents. Curr Pain Headache Rep. 2013; 17: 341.

7. Lebwohl B, Roy A, Alaedini A, Green PH, Ludvigsson JF. Risk of headache-related healthcare visits in patients with celiac disease: A population-based observational study. Headache. 2016; 56: 849-858.

8. Zis $\mathrm{P}$, Julian $\mathrm{T}$, Hadjivassiliou $\mathrm{M}$. Headache associated with coeliac disease: A systematic review and meta-analysis. Nutrients. 2018; 10: 1445.

9. Ameghino L, Farez MF, Wilken M, Goicochea MT. Headache in patients with celiac disease and its response to the gluten-free diet. J Oral Facial Pain Headache. 2019; 33: 294-300. 
10. Bürk K, Farecki ML, Lamprecht $G$, Roth $G$, Decker $P$, Weller $M$, et al. Neurological symptoms in patients with biopsy proven celiac disease. Mov Disord. 2009; 24: 2358-2362.

11. Dimitrova AK, Ungaro RC, Lebwohl B, Lewis SK, Tennyson CA, Green MW, et al. Prevalence of migraine in patients with celiac disease and inflammatory bowel disease. Headache. 2013; 53: 344-355.

12. Assa A, Frenkel-Nir Y, Tzur D, Katz LH, Shamir R. Large population study shows that adolescents with celiac disease have an increased risk of multiple autoimmune and nonautoimmune comorbidities. Acta Paediatr. 2017; 106: 967-972.

13. Roche Herrero MC, Arcas Martínez J, Martínez-Bermejo A, López Martín V, Polanco I, Tendero Gormaz A, et al. The prevalence of headache in a population of patients with coeliac disease. Rev Neurol. 2001; 32: 301-309.

14. Hom GL, Hom BL, Kaplan B, Rothner AD. A single institution's experience of primary headache in children with celiac disease. J Child Neurol. 2020; 35: 37-41.

15. Zelnik N, Pacht A, Obeid R, Lerner A. Range of neurologic disorders in patients with celiac disease. Pediatrics. 2004; 113: 1672-1676.

16. Battistella PA, Mattesi P, Casara GL, Carollo C, Condini A, Allegri F, et al. Bilateral cerebral occipital calcifications and migraine-like headache. Cephalalgia. 1987; 7: 125-129.

17. Serratrice J, Disdier P, de Roux C, Christides C, Weiller PJ. Migraine and coeliac disease. Headache. 1998; 38: 627-628.

18. Morello F, Ronzani G, Cappellari F. Migraine, cortical blindness, multiple cerebral infarctions and hypocoagulopathy in celiac disease. Neurol Sci. 2003; 24: 85-89.

19. D’Amico D, Rigamonti A, Spina L, Bianchi-Marzoli S, Vecchi M, Bussone G. Migraine, celiac disease, and cerebral calcifications: A new case. Headache. 2005; 45: 1263-1267.

20. Benjilali L, Zahlane M, Essaadouni L. A migraine as initial presentation of celiac disease. Rev Neurol. 2012; 168: 454-456.

21. Gabrielli M, Cremonini F, Fiore G, Addolorato G, Padalino C, Candelli M, et al. Association between migraine and celiac disease: Results from a preliminary case-control and therapeutic study. Am J Gastroenterol. 2003; 98: 625-629.

22. Lahat E, Broide E, Leshem M, Evans S, Scapa E. Prevalence of celiac antibodies in children with neurologic disorders. Pediatr Neurol. 2000; 22: 393-396.

23. Inaloo S, Dehghani SM, Farzadi F, Haghighat M, Imanieh MH. A comparative study of celiac disease in children with migraine headache and a normal control group. Turk J Gastroenterol. 2011; 22: 32-35.

24. Balcı O, Yılmaz D, Sezer T, Hızlı Ş. Is celiac disease an etiological factor in children with migraine? J Child Neurol. 2016; 31: 929-931.

25. Alehan F, Ozçay F, Erol I, Canan O, Cemil T. Increased risk for coeliac disease in pediatric patients with migraine. Cephalalgia. 2008; 28: 945-949.

26. Lionetti E, Francavilla R, Maiuri L, Ruggieri $M$, Spina $M$, Pavone $P$, et al. Headache in pediatric patients with celiac disease and its prevalence as a diagnostic clue. J Pediatr Gastroenterol Nutr. 2009; 49: 202-207.

27. Nenna R, Petrarca L, Verdecchia P, Florio M, Pietropaoli N, Mastrogiorgio G, et al. Celiac disease in a large cohort of children and adolescents with recurrent headache: A retrospective study. Dig Liver Dis. 2016; 48: 495-498. 
28. Rossi LN, Cortinovis I, Bellettini G, Brunelli G, Bossi A. Diagnostic criteria for migraine and psychogenic headache in children. Dev Med Child Neurol. 1992; 34: 516-523.

29. Crosato F, Senter S. Cerebral occipital calcifications in celiac disease. Neuropediatrics. 1992; 23: 214-217.

30. Taylor I, Scheffer IE, Berkovic SF. Occipital epilepsies: Identification of specific and newly recognized syndromes. Brain. 2003; 126: 753-769.

31. Bianchi F, Auricchio AM, Battaglia DI, Chieffo DR, Massimi L. Sturge-Weber syndrome: An update on the relevant issues for neurosurgeons. Childs Nerv Syst. 2020; 36: 2553-2570.

32. Kruit MC, van Buchem MA, Launer LJ, Terwindt GM, Ferrari MD. Migraine is associated with an increased risk of deep white matter lesions, subclinical posterior circulation infarcts and brain iron accumulation: The population-based MRI CAMERA study. Cephalalgia. 2010; 30: 129-136.

33. Swartz RH, Kern RZ. Migraine is associated with magnetic resonance imaging white matter abnormalities: A meta-analysis. Arch Neurol. 2004; 61: 1366-1368.

34. Spector JT, Kahn SR, Jones MR, Jayakumar M, Dalal D, Nazarian S. Migraine headache and ischemic stroke risk: An updated meta-analysis. Am J Med. 2010; 123: 612-624.

35. Currie S, Hadjivassiliou M, Clark MJ, Sanders DS, Wilkinson ID, Griffiths PD, et al. Should we be "nervous" about coeliac disease? Brain abnormalities in patients with coeliac disease referred for neurological opinion. J Neurol Neurosurg Psychiatry. 2012; 83: 1216-1221.

36. Hadjivassiliou M, Grünewald RA, Lawden M, Davies-Jones GA, Powell T, Smith CM. Headache and CNS white matter abnormalities associated with gluten sensitivity. Neurology. 2001; 56: 385-388.

37. Ciaccio EJ, Lewis SK, Biviano AB, Iyer V, Garan H, Green PH. Cardiovascular involvement in celiac disease. World J Cardiol. 2017; 9: 652-666.

38. Lebwohl B, Luchsinger JA, Freedberg DE, Green PH, Ludvigsson JF. Risk of dementia in patients with celiac disease: A population-based cohort study. J Alzheimers Dis. 2016; 49: 179-185.

39. Cicarelli G, Della Rocca G, Amboni M, Ciacci C, Mazzacca G, Filla A, et al. Clinical and neurological abnormalities in adult celiac disease. Neurol Sci. 2003; 24: 311-317.

40. Diaconu G, Burlea M, Grigore I, Anton DT, Trandafir LM. Celiac disease with neurologic manifestations in children. Rev Med Chir Soc Med Nat lasi. 2013; 117: 88-94.

41. Jericho $H$, Sansotta $N$, Guandalini S. Extraintestinal manifestations of celiac disease: Effectiveness of the gluten-free diet. J Pediatr Gastroenterol Nutr. 2017; 65: 75-79.

42. Couch JR Jr. Placebo effect and clinical trials in migraine therapy. Neuroepidemiology. 1987; 6: 178-185.

43. Arzani M, Jahromi SR, Ghorbani Z, Vahabizad F, Martelletti $P$, Ghaemi $A$, et al. Gut-brain Axis and migraine headache: A comprehensive review. J Headache Pain. 2020; 21: 15.

44. Mormile R. Celiac disease and migraine: Is there a common backstage? Int J Color Dis. 2014; 29: 1571.

45. Cady RK, Farmer K, Dexter JK, Hall J. The bowel and migraine: Update on celiac disease and irritable bowel syndrome. Curr Pain Headache Rep. 2012; 16: 278-286.

46. Volta U, De Giorgio R, Granito A, Stanghellini V, Barbara G, Avoni P, et al. Anti-ganglioside antibodies in coeliac disease with neurological disorders. Dig Liver Dis. 2006; 38: 183-187. 
47. Volta U, De Giorgio R, Petrolini N, Stangbellini V, Barbara G, Granito A, et al. Clinical findings and anti-neuronal antibodies in coeliac disease with neurological disorders. Scand J Gastroenterol. 2002; 37: 1276-1281.

48. Caio G, De Giorgio R, Venturi A, Giancola F, Latorre R, Boschetti E, et al. Clinical and immunological relevance of anti-neuronal antibodies in celiac disease with neurological manifestations. Gastroenterol Hepatol Bed Bench. 2015; 8: 146-152.

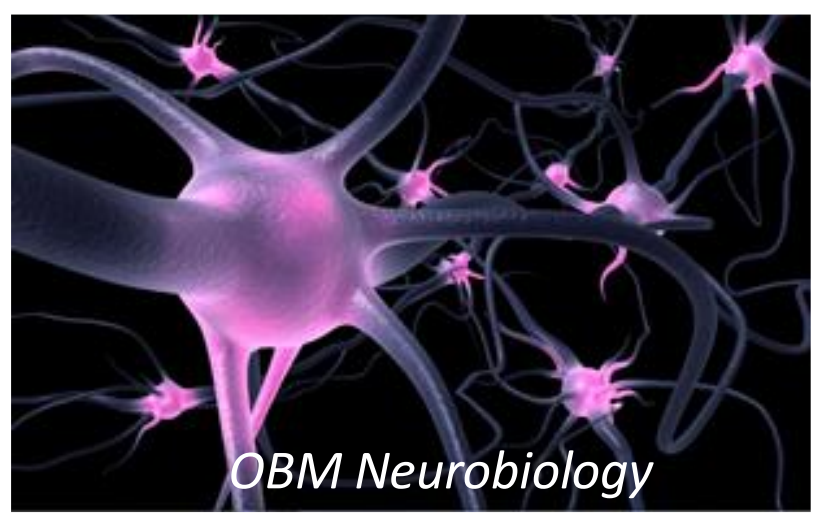

Enjoy OBM Neurobiology by:

1. Submitting a manuscript

2. Joining volunteer reviewer bank

3. Joining Editorial Board

4. Guest editing a special issue

For more details, please visit:

http://www.lidsen.com/journals/neurobiology 